\title{
Hydrothermally generated aromatic compounds are consumed by bacteria colonizing in Atlantis II Deep of the Red Sea
}

\author{
Yong Wang ${ }^{1,2}$, Jiangke Yang ${ }^{1}$, On On Lee ${ }^{1}$, Swagatika Dash ${ }^{1}$, Stanley C.K. Lau ${ }^{1}$, \\ Abdulaziz Al-Suwailem ${ }^{2}$, Tim Y.H. Wong ${ }^{1}$, Antoine Danchin ${ }^{3}$ and Pei-Yuan Qian ${ }^{1}$ \\ ${ }^{1}$ KAUST Global Collaborative Research, Division of Life Science, Hong Kong University of Science and \\ Technology, Hong Kong, China; ${ }^{2}$ King Abdullah University of Science and Technology, Jeddah, Saudi Arabia \\ and ${ }^{3}$ CEA/Genoscope, Evry, France
}

\begin{abstract}
Hydrothermal ecosystems have a wide distribution on Earth and many can be found in the basin of the Red Sea. Production of aromatic compounds occurs in a temperature window of $\sim 60-150{ }^{\circ} \mathrm{C}$ by utilizing organic debris. In the past 50 years, the temperature of the Atlantis II Deep brine pool in the Red Sea has increased from 56 to $68^{\circ} \mathrm{C}$, whereas the temperature at the nearby Discovery Deep brine pool has remained relatively stable at about $44^{\circ} \mathrm{C}$. In this report, we confirmed the presence of aromatic compounds in the Atlantis II brine pool as expected. The presence of the aromatic compounds might have disturbed the microbes in the Atlantis II. To show shifted microbial communities and their metabolisms, we sequenced the metagenomes of the microbes from both brine pools. Classification based on metareads and the 16S rRNA gene sequences from clones showed a strong divergence of dominant bacterial species between the pools. Bacteria capable of aromatic degradation were present in the Atlantis II brine pool. A comparison of the metabolic pathways showed that several aromatic degradation pathways were significantly enriched in the Atlantis II brine pool, suggesting the presence of aromatic compounds. Pathways utilizing metabolites derived from aromatic degradation were also significantly affected. In the Discovery brine pool, the most abundant genes from the microbes were related to sugar metabolism pathways and DNA synthesis and repair, suggesting a different strategy for the utilization of carbon and energy sources between the Discovery brine pool and the Atlantis II brine pool.

The ISME Journal (2011) 5, 1652-1659; doi:10.1038/ismej.2011.42; published online 28 April 2011

Subject Category: geomicrobiology and microbial contributions to geochemical cycles

Keywords: deep sea brine pool; microbes; hydrothermal; aromatic degradation
\end{abstract}

\section{Introduction}

The Red Sea has attracted great attention from geochemists and biologists for its many unique features. Owing to its isolated and regional warm environment, the water of the Red Sea is relatively warm and saline. The sea floor holds many mysteries, including the 25 brine-filled deeps (Swallow and Crease, 1965; Hartmann et al., 1998). Geochemical studies have shown most of these deeps to be hypersaline and metalliferous brine pools (Gurvich, 2006).

Since their discovery in 1965 (Swallow and Crease, 1965), two deep-sea brine pools—the Discovery Deep and Atlantis II Deep-have been the

Correspondence: P-Y Qian, Department of Biology, Division of Life Science, Hong Kong University of Science and Technology, KAUST Global Collaborative Research, Clear Water Bay, Hong Kong, China.

E-mail: boqianpy@ust.hk

Received 26 November 2010; revised 7 February 2011; accepted 16 March 2011; published online 28 April 2011 focus of geologists studying the Red Sea. The temperature, salinity and metal content of the two brine pools have been extensively surveyed. The short geographic distance and parallel changes in anhydrite in the sediment pore water indicate that the two brine pools were connected historically (Swallow and Crease, 1965; Monnin and Ramboz, 1996). However, the temperature in the Atlantis II Deep increased from $56^{\circ} \mathrm{C}$ in 1965 to $71^{\circ} \mathrm{C}$ in 1995 (Swallow and Crease, 1965; Hartmann et al., 1998), whereas that of the Discovery Deep has remained fairly constant. The gradual increase in temperature in Atlantis II Deep and additional geographical data strongly suggest that the two systems are now separate (Miller et al., 1966; Ross and Hunt, 1967). The temperature increase in Atlantis II Deep is the result of a flux of hot brine in the temperature range of $195-310^{\circ} \mathrm{C}$ from the bottom of the pool (Anschutz and Blanc, 1996). Perhaps because of this flux, the Atlantis II Deep brine pool (ABP) consists of a lower convective layer and three upper convective layers that differ in salinity and temperature (Blanc and 
Anschutz, 1995). The high temperature, together with a high water pressure and metal content, means that the ABP is a typical hydrothermal system (Winckler et al., 2000). The hydrothermal effects to which it is subjected trigger reduction reactions that produce aromatic compounds from organic debris. The temperature window for aromatic compound formation is $\sim 60-150{ }^{\circ} \mathrm{C}$, beyond which debris is degraded to methane (Simoneit, 1992). Recent studies have reported a fourfold higher abiogenic methane content in ABP than in the Discovery Deep brine pool (DBP), implying that the high temperature of the hot brine flux and deep sediment in ABP are the main geological factors inducing the release of methane into the brine pool (Faber et al., 1998). In contrast, the DBP keeps a nearly constant temperature of $40-44^{\circ} \mathrm{C}$ (Hartmann et al., 1998), serving as an analog of the ancestral ecosystem of ABP. The presence of aromatic compounds in DBP is not expected because its temperature is below the theoretical threshold temperature for producing the compounds.

Hypothetically, as the temperature gap between the two brine pools widens, the concentration of aromatic compounds should differ more sharply. Accordingly, aromatic-degrading bacteria should be enriched and both the bacterial community and metabolic profile of ABP should be altered. To test this hypothesis, we studied the microbial communities of the two ecosystems and found evidence of the influence of hydrothermal disturbance on the microbial inhabitants. A comparison of the bacterial composition of the two pools and the metabolic pathways of the bacterial inhabitants thus sheds light on the history of change in the Atlantis II Deep hydrothermal ecosystem.

\section{Materials and methods}

\section{Sampling}

In October 2008, brine water samples were collected from the ABP $\left(21^{\circ} 20.76^{\prime} \mathrm{N}, 38^{\circ} 04.68^{\prime} \mathrm{E}\right)$ and DBP $\left(21^{\circ} 16.99^{\prime} \mathrm{N}, 38^{\circ} 03.05^{\prime} \mathrm{E}\right)$ during the R/V OCEANUS cruise (Bower, 2009). The depth of the sampling sites was $>2100 \mathrm{~m}$. At each sampling location, $40 \mathrm{l}$ of brine water were collected from a hydrocast equipped with four $10 \mathrm{l}$ Niskin bottles connected in series. Temperature and other environmental parameters such as pressure and salinity were recorded by a CTD (conductivity, temperature and depth) unit. The brine water temperature at the sampling site was $68^{\circ} \mathrm{C}$ for the $\mathrm{ABP}$ and $45^{\circ} \mathrm{C}$ for the DBP (Bower, 2009). The samples were immediately filtered through a 1.6- $\mu \mathrm{m} \mathrm{GF} / \mathrm{A}$ membrane (diameter $125 \mathrm{~mm}$; Whatman, Clifton, NJ, USA) to remove suspended particles and diatoms, and then through a $0.22-\mu \mathrm{m}$ polycarbonate membrane (diameter $45 \mathrm{~mm}$; Millipore, Bedford, MA, USA) to capture microbial cells. The $0.22-\mu \mathrm{m}$ polycarbonate membrane was then frozen at $-80^{\circ} \mathrm{C}$ in $0.8 \mathrm{ml}$ of extraction buffer ( $40 \mathrm{mM}$ of EDTA, $0.75 \mathrm{M}$ of sucrose, $0.5 \mathrm{M}$ of $\mathrm{NaCl}, 50 \mathrm{mM}$ of Tris; $\mathrm{pH}$ 8). Total genomic DNA was extracted and purified according to the SDS-based method described elsewhere (Huber et al., 2002). The purified DNA was then dissolved in $50 \mu \mathrm{l}$ of double-distilled $\mathrm{H}_{2} \mathrm{O}$ and stored at $-20^{\circ} \mathrm{C}$ until use.

\section{Chemical analysis of the brine water samples}

Brine water samples ( $500 \mathrm{ml}$ each) from the ABP and DBP were filtered using a $20-\mu \mathrm{m}$ filter (Millipore). The filter was subjected to extraction three times with $15 \mathrm{ml}$ of methanol under sonication, and the extracts were combined and dried under a vacuum. The filtrate was freeze dried and extracted with methanol. Both extracts were concentrated at a reduced pressure and then loaded onto five Seppak C18 cartridges (Waters, Milford, MA, USA) followed by washing with Milli-Q water and eluted with $100 \%$ methanol and then with $100 \%$ acetonitrile. The extracts were further concentrated under reduced pressure and partitioned with 1:1 dichloromethane and methanol. The non-polar dichloromethane fraction was subjected to gas chromatography-mass spectrometry (GC-MS) analysis. Polar organic compounds bearing hydroxyl and carboxylic groups present in the methanol fraction were derived using N,O-bis(trimethylsilyl)trifluoroacetamide and identified by GC-MS.

GC was performed on relatively non-polar capillary columns (CP-Sil 8 CB-MS, 30-m length, 0.25- $\mu \mathrm{m}$ film thickness, $0.25 \mathrm{~mm}$ in diameter; Varian 3800 , Palo Alto, CA, USA). The GC oven was initially held at $80^{\circ} \mathrm{C}$ for $5 \mathrm{~min}$, heated at increments of $3{ }^{\circ} \mathrm{Cmin}^{-1}$ to $200{ }^{\circ} \mathrm{C}$ and held for $2 \mathrm{~min}$, then increased to $300{ }^{\circ} \mathrm{C}$ at $15^{\circ} \mathrm{Cmin}^{-1}$. The injector, the ion trap and the interface temperatures were set to $275^{\circ} \mathrm{C}, 200^{\circ} \mathrm{C}$ and $300{ }^{\circ} \mathrm{C}$, respectively. Metabolites were identified and then verified by comparison with the NIST database (http://srdata.nist.gov/) and standards. The relative area of the peaks in the base-peak chromatogram was used for quantitative comparison ( $\mathrm{Hu}$ et al., 2008).

\section{$16 S$ rRNA gene clone library construction and} sequencing

Two primers, 27F ( $5^{\prime}$-AGAGTTTGATCCTGGCTCA G-3') and 1055R ( $5^{\prime}$-CACGAGCTGACGACAGCCAT-3'), were used to amplify bacterial $16 \mathrm{~S}$ rRNA gene fragments with a Bio-Rad (Hercules, CA, USA) thermal cycler using the following cycling conditions: initial denaturation at $94{ }^{\circ} \mathrm{C}$ for $5 \mathrm{~min}$; 28 cycles of $94{ }^{\circ} \mathrm{C}$ for $50 \mathrm{~s}, 55^{\circ} \mathrm{C}$ for $50 \mathrm{~s}$ and $72^{\circ} \mathrm{C}$ for $50 \mathrm{~s}$; and final extension at $72^{\circ} \mathrm{C}$ for $6 \mathrm{~min}$ (Lee et al., 2009). The PCR products from three reactions were pooled to minimize bias, and purified by using a gel purification kit (Qiagen, Hilden, Germany). The purified PCR products were cloned into pCR2.1TOPO vectors and transformed into TOP10 
competent cells (Invitrogen, Carlsbad, CA, USA). White colonies were randomly placed in a 96-well plate containing lysogeny broth (LB) $\left(100 \mu \mathrm{g} \mathrm{ml}^{-1}\right.$ of ampiciline), and then sequenced by using an ABI 3730 automatic sequencer (Applied BioSystems, Foster City, CA, USA) with M13F and M13R primers. In total, 117 clone sequences were obtained for the ABP and 119 for the DBP. The genera and their percentages are shown in Supplementary Figure S1. The clone library sequences for $A B P$ and DBP were compared in the RDP database (http:// rdp.cme.msu.edu/comparison/). The result is shown in Supplementary Table S1.

\section{4 pyrosequencing}

As the total crude DNA did not meet the minimal requirement for 454 pyrosequencing, a Repli-g Whole Genome Amplification kit (Qiagen) was used to amplify the microbial genomic DNA from the two brine pools. Amplified DNA was subjected to pyrosequencing on a ROCHE (Nutley, NJ, USA) 454 FLX Titanium platform at the Chinese National Human Genome Center in Shanghai (China). The 454 Titanium platform generated about 990000 and 910000 reads with an average length of 396 and $413 \mathrm{bp}$ for the ABP and DBP samples, respectively.

Detecting rRNA fragments from the metareads rRNA HMM (Huang et al., 2009) was used to identify the 16S rRNA fragments from the 454 reads using the default settings. A total of 823 and 477 rRNA fragments were extracted from the data sets and filtered by using the RDP database (10.9) (Cole et al., 2009). After the removal of short fragments that could not be classified, 792 fragments were retained for the $\mathrm{ABP}$ and 453 fragments for the DBP. The best matches were used to assign taxonomic affiliations to the fragments at the phylum and genus levels.

\section{Identification of Kyoto Encyclopedia of Genes and Genomes (KEGG) genes}

The KEGG protein database (v51) (Allen and Banfield, 2005), which encompasses protein sequences from 930 archaeal and bacterial strains, was downloaded. Pyrosequencing reads were used to blast against the two protein databases using the parameters '- $p$ blastx -v 5-b 5-e $0.0001-\mathrm{m}$ 8-Q 11'. The results of the blasts were summarized and the best hits were selected to represent the KEGG genes identified from the reads. The frequencies of the KEGG genes were recorded and used to rank the genes.

Clustering the KEGG genes with Global Ocean Sampling (GOS) reference samples

GOS 17, 30 and 33 shotgun sequences were downloaded from CAMERA (Seshadri et al., 2007). The GOS sequences were split into $450 \mathrm{bp}$ fragments.
The same Blast parameters were used to identify the KEGG prokaryotic genes. The frequencies of the genes were converted to percentages for each of the samples and for each brine pool. The three reference data sets, together with the two brine pool data sets, were put into Cluster 3 (Eisen et al., 1998), and 548 genes with a percentage interval of at least $1 \%$ between any two samples were retained. Their percentages were further normalized and clustered using the complete linkage method and a metric of correlation (uncentered).

The KEGG genes specifically over-represented in the GOS, ABP and DBP were extracted from the clusters and packed into pathway maps to show their roles in metabolic and functional pathways. Maps showing the most frequent KEGG genes present with their EC (Enzyme Commission) numbers or KEGG annotations were then generated. The file containing the linkage between the EC numbers and the maps can be downloaded from the KEGG. Each map had a minimum of four nodes (genes or cofactors), at least one of which was not shared by the other maps. If not all of the nodes in a map were unique, then the map had to have at least nine nodes.

KEGG pathways showing significant differences in map completeness

To further compare the metabolic profiles of the ABP and DBP, maps showing significant differences in completeness were generated. Among the KEGG genes, over-represented genes occurring at a greater frequency than the average for all of the genes were selected. For the ABP, 960 genes with more than 18 occurrences were selected, and for the DBP, 970 genes with more than 41 occurrences were selected. The genes were ranked according to their frequencies. The top $10 \%$ of the genes were then picked out and assigned to maps. The number of nodes in a map was recorded for both samples. The top $20 \%$ of all the ranked genes were then used to calculate the number of nodes. A $10 \%$ gene increment was applied to obtain 10 pairs of node numbers for individual pathway maps of each brine pool. A Mann-Whitney test was used to compare the two sets of node numbers and to show the maps that differed significantly $(P<0.05)$ in completeness between the two brine pools. In all, 32 KEGG maps that had more genes (cofactors) in either the ABP or DBP were obtained. The activity of a pathway was indicated by the completeness of its map.

\section{Results and discussion}

Discovery of aromatic compounds in Atlantis II Deep To verify the presence of aromatic compounds in the $\mathrm{ABP}$, we analyzed the brine water with GC-MS analysis. At least five aromatic compounds with basic aromatic structures of benzoic acid and phenol were identified (Figure 1 and Supplementary Table 
<smiles>CCOC(=O)OCCOc1ccc(C(=O)OCC)cc1</smiles><smiles>Cc1c(O)cc(C(F)(F)F)c(O)c1C</smiles>

Phen-1,4-diol, 2,3-dimethyl-5-trifluoromethyl-

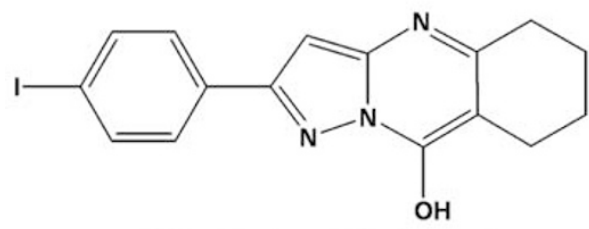

2-(4-lodo-phenyl)-5,6,7,8-tetrahydropyrazolo[5,1-b]quinazolin-9-ol<smiles>O=C(O)c1ccccc1C(=O)O</smiles>

1,2-Benzenedicarboxylic acid

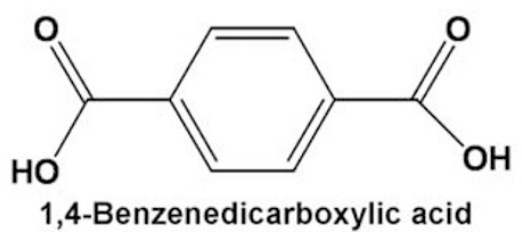

Figure 1 Aromatic compounds found in the ABP. The structures of the compounds were predicted on the basis of the data generated by the GC-MS analysis.

S2). Although we did not use the standards to verify their structures and accurately quantify the concentrations, on the basis of the peak area and height that also can be proportional to abundance of substance present in the sample, the compound 2-(4-Iodophenyl)-5,6,7,8-tetrahydro-pyrazoloquinazolin-9-ol was highly abundant in the ABP. In contrast, neither these nor any other aromatic compounds were detected in the DBP. Instead, we detected low concentrations of alcohols and esters in the DBP.

The hydrothermally generated aromatic compounds have been also detected in the Kebrit and Shaban Deeps in the Red Sea (Michaelis et al., 1990). However, perhaps because of poor detection sensitivity then, the presence of the aromatic compounds in the ABP was not confirmed in a study conducted in 1987 (Simoneit et al., 1987).

Different microbial communities in the two brine pools The microbes in the two brine pools were then studied to demonstrate the predicted shift of microbial communities and the divergence of their metabolisms for adaptation. We obtained nearly one million of metareads with an average length of about $400 \mathrm{bp}$ from the two samples. Genes in the reads were searched by Blasting against the KEGG database (Kanehisa and Goto, 2000). We identified fragments of the $16 \mathrm{~S}$ rRNA genes from the metareads and assigned them into phyla and genera as a preliminary survey of the bacteria living in the two pools (Supplementary Tables S3 and S4). The results showed differences in bacterial composition. Cupriavidus was dominant in both brine pools, accounting for $50 \%$ of 789 and $43 \%$ of 223 bacterial fragments in the ABP and DBP, respectively (Figure 2). Bacteria in this genus are generally found in metalliferous environments, and the prevalence of this genus in our samples is thus reasonable (Vandamme and Coenye, 2004). Likewise, the high percentage of the metal-resistant genera Wautersia and Ralstonia is also unsurprising (Nies, 2000). The genomes and inner plasmids of species in these genera contain numerous metal-resistant genes, which support the findings from the metareads.

However, the two samples differed remarkably in the presences of aromatic degrading genera. Acinetobacter, Mycobacterium and Alkanindiges accounted for a total of $18.2 \%$ of all of the $16 \mathrm{~S}$ rRNA fragments in the ABP sample, which is almost 10 times higher than their proportion in the fragments from the DBP (Figure 2). Bacteria in these genera are mostly found in natural soil and water (Prince et al., 2010). Their spreading in the ABP may explain the high occurrence of genes for aromatic degradation and confirm the presence of aromatic compounds in the pool. In addition, both Cupriavidus and Ralstonia are able to utilize aromatic compounds from the environment (Lykidis et al., 2010). Undescribed bacterial inhabitants included small populations of Bradyrhizobium (2.4\%) and Phyllobacterium $(1.5 \%)$, which are involved in nitrogen fixation (Pate, 2002). The main composition of the microbial communities in the two samples was confirmed by classifying the clone sequences of bacterial full-length 16S rRNA genes (Supplementary Figure S1). The percentages of the dominant species were largely consistent with those previously identified from the metareads, although some minor genera, such as Alkanindiges, were not detected in the clone sequences (Supplementary 


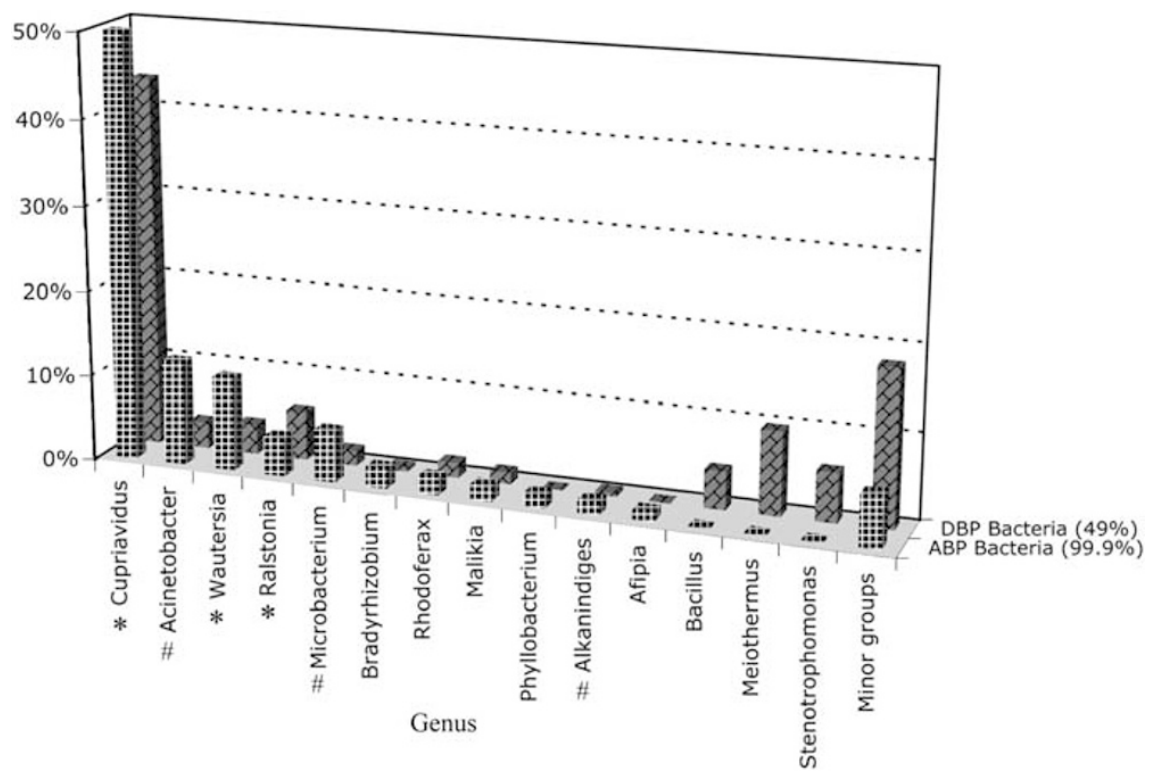

Figure 2 Bacterial genera in the Atlantis II Deep and Discovery Deep brine pools. Bacterial composition in the Atlantis II Deep (ABP) and Discovery (DBP) Deep brine pools was estimated by taxonomically classifying the 16S rRNA fragments identified from metadata reads. Classification at the genus level was performed by using the RDP classifier (Cole et al., 2009). No confidence threshold was applied to the classification, and the best match in the RDP database was used to determine the genus of a fragment. The percentages of bacterial 16S rRNA fragments are shown in parentheses. The minor groups refer to a collection of genera comprising $<1 \%$ of the total $16 \mathrm{~S}$ rRNA

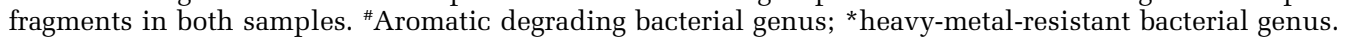

Figure S1). Further, some genera identified using the clone library were absent from the metareads. Although the differences at the genus level between the two samples can in large part be explained by the different compositions of the bacterial communities, the influence of sampling drift should not be ignored. The estimated microbial cell density in our samples was 9200 cells ml $^{-3}$ for the ABP and 7000 cells $\mathrm{ml}^{-3}$ for the DBP. These low densities may lead to sampling drift and the subsequent inaccurate characterization of the structures of the bacterial communities. However, although minor genera were absent in some of the samples, the major genera were successfully detected despite their differing proportions in the two communities.

The archaeal $16 \mathrm{~S}$ fragments could not be reliably classified into genera by referring to the present collection of the RDP database (data not shown). Archaea (mostly Euryarchaeota) accounted for about $50 \%$ of the whole community of the DBP, and could be methanogens. On the contrary, we found only three $16 \mathrm{~S}$ fragments for Archaea (Crenarchaeota) in the ABP. Although Archaea are usually thermotolerant, the small archaeal population in the ABP indicates that some unknown limiting factors for them to flourish in the hydrothermal system may exist.

\section{Enrichment of aromatic degradation pathways in the $A B P$}

A total of 3080 and 3999 KEGG genes were retrieved from the ABP and DBP, respectively, and their frequencies were recorded (ABP: 55029 copies; DBP: 153722 copies). It was expected that the frequency and metabolic pathway of the genes encoded by the bacteria in the brine pools would be different in response to the different environments and chemical sources in the two pools. We thus focused on abundant genes and their functions in the metareads of the two pools.

To show the differences in gene content between the brine pool systems and other aquatic systems, the ABP and DBP samples were compared with three reference samples: GOS 17, 30 and 33 collected during the GOS expedition (Rusch et al., 2007). The KEGG genes with the largest difference in frequency, some 548 genes in total, were used to show the similarity between the samples. The clustering of the KEGG genes showed a closer relationship between the ABP and DBP brine samples (Figure 3). The GOS 17, 30 and 33 samples were obtained from surface water and, not surprisingly, displayed a greater similarity in gene content. GOS 33 was sampled from the surface water of a lake with a high salinity of $63.4 \%$ and a high temperature of $38^{\circ} \mathrm{C}$. The stress from these two physical factors may partially explain the similarity in gene profiles observed between GOS 33 and the two brine pools, which are both hypersaline and hyperthermal (Figure 3). The two brine pools are similar to each other in many respects, but the clustering method allowed the visualization of sample-specific gene clusters (Figure 3). A large fraction of the KEGG genes from the DBP-specific cluster encoded transposases and metal ion pumps, whereas the ABP-specific cluster contained genes responsible for the degradation of aromatic compounds (Supplementary Tables S5 and S6). 


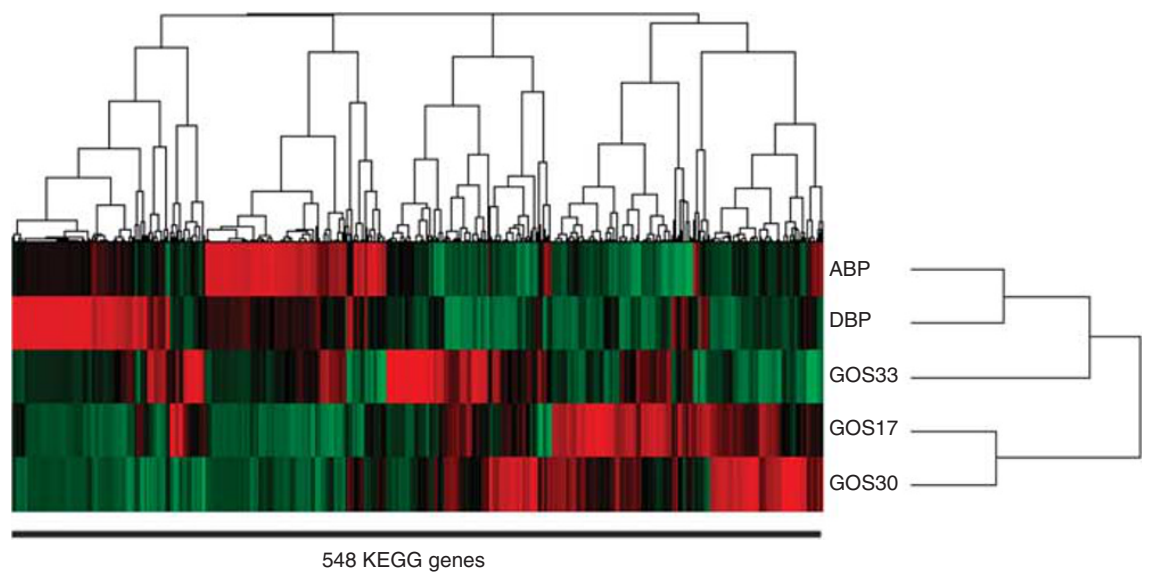

Figure 3 Clustering of KEGG genes from the ABP and DBP in reference to three GOS data sets. Percentage of KEGG genes in the ABP and DBP metadata compared with those in the three reference GOS data sets. Only genes showing a $>1 \%$ difference were used for the clustering. The color code indicates the relative abundance of the genes, ranging from green (low abundance) via black to red (high abundance).

To further verify the metabolic roles of the enriched KEGG genes in the two samples (that is, those occurring at a greater frequency than the average for all of the genes), we placed the enriched genes into KEGG pathway maps. The number of nodes in the 32 resulting maps differed significantly between the two samples (Mann-Whitney test; $P<0.05$ ), and the significance of the difference increased with the number of genes involved in the analysis (Figure 4). The number of nodes reflects the completeness of each map, and an active KEGG pathway contains more nodes. The pathways that were particularly active in the ABP were related to $\gamma$-hexachlorocyclohexane degradation (ko00361), benzoate degradation (ko00362 and ko00632), 1- and 2-methylnaphthalene degradation (ko00626), caprolactam degradation (ko00930), and limonene and pinene degradation (ko00903). Taken together with the findings shown in Figure 3, the maps show that the aromatic compounds involved in these degradation pathways and other similar compounds are probably hydrothermally produced, biologically degraded and utilized by microbes in the ABP as carbon sources. The temperature required for such reduction reactions is $\sim 60-150^{\circ} \mathrm{C}$ (Simoneit, 1992). In the last few decades, the temperature in the ABP has risen gradually to $68^{\circ} \mathrm{C}$, which is above the lower threshold. Our findings thus lend support to the geochemical theory that this is the threshold temperature for the formation of aromatic compounds. The microbiological evidence further shows that hydrothermal conditions somehow disturb the ecosystem in the pools, as reflected by the differences in the bacterial communities and metabolic pathways for the degradation of aromatic compounds in the ABP and DBP.

The impact of hydrothermal effects is also reflected by the differences in the pathways involved in the utilization of metabolites derived from aromatic-compound degradation between the two pools. According to the KEGG maps, the complex aromatic compounds were first biochemically degraded to benzoate and then further degraded via CoA ligation and hydroxylation for downstream processes of the citrate cycle, pyruvate and butanoate metabolism, and glycolysis (van der Meer, 1997). The derivatives from such degradation processes can be used in a variety of pathways, such as aromatic amino acid metabolism and fatty acid metabolism. Correspondingly, fatty acid biosynthesis and metabolism (ko00061 and ko00071); valine, leucine and isoleucine degradation (ko00280); tyrosine metabolism (ko00350); phenylalanine metabolism (ko00360); and glutathione metabolism (ko00480) were more abundant in the ABP (Figure 4). The GC-MS results revealed the presence of several fatty acids in the ABP brine pool too, including decanal dimethyl acetal, butanedioic acid, hexanedioic acid and hexadecanoic acid (Supplementary Figure S2 and Supplementary Table S1). In contrast, the DBP brine showed enriched aromatic biosynthesis pathways, with significantly more complete pathways for lysine biosynthesis (ko00300); histidine metabolism (ko00340); phenylalanine, tyrosine and tryptophan biosynthesis (ko00400); thiamine metabolism (ko00730); pantothenate and CoA biosynthesis (ko00770); porphyrin and chlorophyll metabolism (ko00860); and terpenoid biosynthesis (ko00900). The DBP bacteria probably utilize sugar as carbon and energy sources, as indicated by the enrichment of sugar pathways, including glycolysis/gluconeogenesis (ko00010), the pentose phosphate pathway (ko00030), and starch and sucrose metabolism (ko00500) (Figure 4).

In addition to the discovery of aromatic degradation pathways, we found more chemotactic genes in the ABP. For example, the bottom flux of saline water in the salinity range of $270-370 \%$ (Anschutz and Blanc, 1996) creates a large halocline in the ABP. More genes with motility and chemotaxis functions were identified in the KEGG genes from 


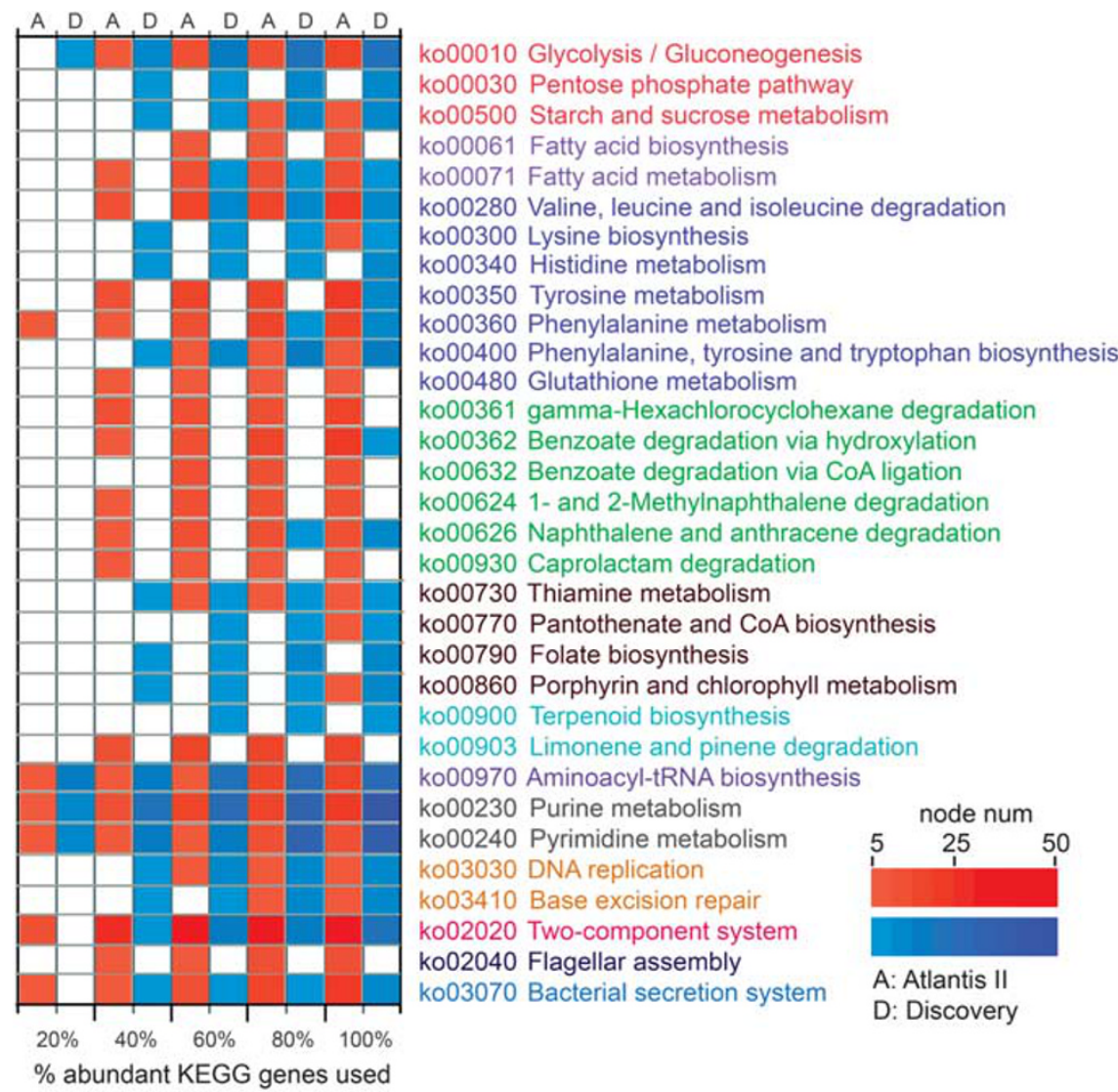

Figure 4 KEGG maps showing significant differences in completeness between the ABP and DBP. Enriched KEGG genes (those with a copy number larger than the average number of all of the genes) were placed in KEGG maps with their EC numbers or KEGG annotations. Genes were ranked based on copy number. The top 10\% of abundant genes were included in the pathway maps, and the node number of the maps recorded. Using a $10 \%$ increment of the top-ranking genes, 10 sets of node numbers for each KEGG map were obtained. The number of nodes in the Atlantis II (A) and Discovery (D) brine pools was compared using a Mann-Whitney $U$-test. The figure shows a map of the significant differences between the two samples $(P<0.05)$. A KEGG map has many nodes, each of which represents a gene or cofactor. The increase in the number of nodes using a $20 \%$ gene increment is indicated by the color code. A white box indicates zero nodes. The names of KEGG pathways in the same functional categories are displayed in the same color.

the ABP metareads. They were concentrated in the pathways of the two-component system (ko02020), flagellar assembly (ko02040) and the bacterial secretion system (ko03070) (Figure 4). Their presence may provide an adaptive advantage to the microbes in ABP. However, we could neither confirm whether they were original genes from the aromatic degraders nor could we determine the expression level of these genes in the ABP. If they are abundant in the genomes of aromatic compound-degrading bacteria, but largely inactive after colonization in the ABP, then the overrepresentation of the chemotactic genes is not likely to be a result of adaptation.

\section{Concluding remarks}

In conclusion, we compared the metabolic pathways and bacterial communities in two famous deep-sea brine pools in the Red Sea. The occurrence of bacterial species capable of degrading aromatic compounds, the presence of abundant aromatic and downstream metabolic pathways, and the successful detection of several aromatic compounds in the ABP provide biological and chemical evidence for geochemical theory on the production of aromatic compounds under hydrothermal conditions. However, in this study, we could not measure the concentrations of the aromatic compounds and did not obtain the pure bacterial strains (although different culture media were used) for examining the bacterial capacity of degrading aromatic compounds. The differences in the microbial compositions between the pools might not be totally attributed to the production of the aromatic compounds. The lack of Archaea in the ABP could serve as a possible example. Therefore, the other causal factors affecting the microbial communities and altering genomic features in the two brine pools shall deserve further investigations.

Considering the increasing hydrothermal activities occurring in ABP, we advocate regular monitoring of the dynamics of the microbial communities in these hydrothermal systems. Our ongoing effort is to analyze the composition of organic compounds in different layers and sediments in the ABP to better understand the 
development of adaptive mechanisms in response to changing environmental conditions.

\section{Conflict of interest}

The authors declare no conflict of interest.

\section{Acknowledgements}

We are grateful to Professor Roberto Kolter for his constructive comments on this manuscript, the captain and crew of the R/V Oceanus for providing technical help during the sampling, and A Bower and S Swift of the Woods Hole Oceanographic Institution for providing environmental data and useful information about the study sites. This publication is on the basis of the work supported by an award (SA-C0040/UK-C0016) made by KAUST to PY Qian.

\section{References}

Allen E, Banfield J. (2005). Community genomics in microbial ecology and evolution. Nat Rev Microbiol 3: 489-498.

Anschutz P, Blanc G. (1996). Heat and salt fluxes in the Atlantis II Deep (Red Sea). Earth Planet Sci Lett 142: 147-159.

Blanc G, Anschutz P. (1995). New stratification in the hydrothermal brine system of the Atlantis II Deep, Red Sea. Geology 23: 543-546.

Bower AS. R/V Oceanus Voyage 449-6 Red Sea Atlantis II Deep Complex Area 19 October-1 November 2008. Woods Hole Oceanographic Institution, 2009.

Cole JR, Wang Q, Cardenas E, Fish J, Chai B, Farris RJ et al. (2009). The Ribosomal Database Project: improved alignments and new tools for rRNA analysis. Nucleic Acids Res 37: 141-145.

Eisen MB, Spellman PT, Brown PO, Botstein D. (1998). Cluster analysis and display of genome-wide expression patterns. Proc Natl Acad Sci USA 95: 14863-14868.

Faber E, Botz R, Poggenburg J, Schmidt M, Stoffers P, Hartmann M. (1998). Methane in Red Sea brines. Org Geochem 29: 363-379.

Gurvich EG. (2006). Metalliferous sediments of the Red Sea. Metalliferous Sediments of the World Ocean. Springer: Berlin, Heidelberg.

Hartmann M, Scholten JC, Stoffers P, Wehner F. (1998). Hydrographic structure of brine-filled deeps in the Red Sea-new results from the Shaban, Kebrit, Atlantis II, and Discovery Deep. Mar Geol 144: 311-330.

Hu D, Bian Q, Li TWY, Lau AKH, Yu JZ. (2008). Contributions of isoprene, monoterpenes, caryophyllene, and toluene to secondary organic aerosols in Hong Kong during the summer of 2006. J Geophys Res 113: D22206.

Huang Y, Gilna P, Li W. (2009). Identification of ribosomal RNA genes in metagenomic fragments. Bioinformatics 25: $1338-1340$.

Huber JA, Butterfield DA, Baross JA. (2002). Temporal changes in archaeal diversity and chemistry in a mid-ocean ridge subseafloor habitat. Appl Environ Microbiol 68: 1585-1594.

Kanehisa M, Goto S. (2000). KEGG: Kyoto Encyclopedia of Genes and Genomes. Nucleic Acids Res 28: 27-30.

Lee OO, Wong YH, Qian P-Y. (2009). Inter- and intraspecific variations of bacterial communities associated with marine sponges from San Juan island, Washington. Appl Environ Microbiol 75: 3513-3521.

Lykidis A, Parez-Pantoja D, Ledger T, Mavromatis K, Anderson IJ, Ivanova NN et al. (2010). The complete multipartite genome sequence of Cupriavidus necator JMP134, a versatile pollutant degrader. PLOS ONE 5: e9729.

Michaelis W, Jenisch A, Richnow HH. (1990). Hydrothermal petroleum generation in Red Sea sediments from the Kebrit and Shaban deeps. Appl Geochem 5: 103-114.

Miller AR, Densmore CD, Degens ET, Hathaway JC, Manheim FT, McFarlin PF et al. (1966). Hot brines and recent iron deposits in deeps of the Red Sea]. Geochim Cosmochim Acta 30: 341-350.

Monnin C, Ramboz C. (1996). The anhydrite saturation index of the ponded brines and sediment pore waters of the Red Sea deeps. Chem Geol 127: 141-159.

Nies DH. (2000). Heavy metal-resistant bacteria as extremophiles: molecular physiology and biotechnological use of Ralstonia sp. CH34. Extremophiles 4: 77-82.

Pate JS. Symbiotic nitrogen fixation between microorganisms and higher plants of natural ecosystems. In: Microorganisms in Plant Conservation and Biodiversity. Kluwer Academic Publishers: New York, 2002, pp 45-77.

Prince RC, Gramain A, McGenity TJ. (2010). Handbook of Hydrocarbon and Lipid Microbiology. Springer: Berlin.

Ross DA, Hunt JM. (1967). Third brine pool in the red sea. Nature 213: 687-688.

Rusch DB, Halpern AL, Sutton G, Heidelberg KB, Williamson S, Yooseph S et al. (2007). The Sorcerer II Global Ocean Sampling Expedition: northwest Atlantic through eastern tropical Pacific. PLoS Biol 5: e77.

Seshadri R, Kravitz SA, Smarr L, Gilna P, Frazier M. (2007). CAMERA: a community resource for metagenomics. PLoS Biol 5: e75.

Simoneit BRT. (1992). Aqueous organic geochemistry at high temperature/high pressure. Origins of Life and Evolution of the Biosphere. Kluwer Academic Publishers: New York, pp 43-65.

Simoneit BRT, Grimalt JO, Hayes JM, Hartman H. (1987). Low temperature hydrothermal maturation of organic matter in sediments from the Atlantis II Deep, Red Sea]. Geochim Cosmochim Acta 51: 879-894.

Swallow JC, Crease J. (1965). Hot salty water at the bottom of the Red Sea. Nature 205: 165-166.

Vandamme P, Coenye T. (2004). Taxonomy of the genus Cupriavidus: a tale of lost and found. Int J Syst Evol Microbiol 54: 2285-2289.

van der Meer JR. (1997). Evolution of novel metabolic pathways for the degradation of chloroaromatic compounds. Antonie van Leeuwenhoek 71: 159-178.

Winckler G, Kipfer R, Aeschbach-Hertig W, Botz R, Schmidt M, Schuler S et al. (2000). Sub sea floor boiling of Red Sea brines: new indication from noble gas data]. Geochim Cosmochim Acta 64: 1567-1575.

Supplementary Information accompanies the paper on The ISME Journal website (http://www.nature.com/ismej) 